\title{
Effect of K substitution on Magnetoresistivity and activation energy of Bi-2212 system
}

\author{
B. Özçelik ${ }^{a^{*}}$, E.Yalaz $^{\mathrm{a}}$, M.E.Yakıncı ${ }^{\mathrm{b}}$, A. Sotelo ${ }^{\mathrm{c}}$, M. A. Madre ${ }^{\mathrm{c}}$ \\ ${ }^{a}$ Department of Physics, Faculty of Sciences and Letters, Çukurova University. 01330 Adana, \\ Turkey \\ ${ }^{b}$ Department of Physics, Faculty of Sciences and Letters, Inönü University 44069, Malatya, \\ TURKEY \\ ${ }^{c}$ ICMA (CSIC-Universidad de Zaragoza). María de Luna, 3. 50018 Zaragoza, Spain.
}

\begin{abstract}
In the present work, magnetoresistivity performance of polycrystalline $\mathrm{Bi}_{2} \mathrm{Sr}_{2} \mathrm{Ca}_{1-\mathrm{x}} \mathrm{K}_{\mathrm{x}} \mathrm{Cu}_{2} \mathrm{O}_{8+\mathrm{y}}$ superconductor with $\mathrm{x}=0.0,0.05,0.075$, and 0.1 has been studied by the change of the flux pinning mechanism using magnetoresistivity measurements. The samples were prepared by using a polymer solution technique. The effects of $\mathrm{K}$ substitution for $\mathrm{Ca}$ on the irreversibility field, upper critical magnetic field, coherence length and activation energies have been investigated. The magnetoresistivity of the samples has been measured under applied magnetic fields ranging from 0 to 9 Tesla. Broadening of superconducting transition is observed under magnetic field, explained on the basis of Thermally Activated Flux Flow (TAFF) model. The upper critical magnetic field $H_{c 2}(0)$ and the coherence length $(\zeta(0))$ at $T=0 \mathrm{~K}$ were calculated using the resistivity data and from $H_{c 2}(0)$, respectively. $H_{C 2}(0)$ and $\xi(0)$ values have been calculated as $62,72,75,53$ and $23,21,20,25$, for the $0.0,0.05,0.075$, and $0.10 \mathrm{~K}$ doped samples, respectively. TAFF model has been studied in order to calculate the flux pinning energies. In particular, the flux pinning energies of $\mathrm{Bi}_{2} \mathrm{Sr}_{2} \mathrm{Ca}_{1-\mathrm{x}} \mathrm{K}_{\mathrm{x}} \mathrm{Cu}_{2} \mathrm{O}_{8+\mathrm{y}}$ where $\mathrm{x}=0.075$ are calculated as $0.0147 \mathrm{eV}$ for $9 \mathrm{~T}$ and $0.29 \mathrm{eV}$ for $0 \mathrm{~T}$.
\end{abstract}

Keywords: High- $T_{C}$ superconductors, Magnetoresistivity, Activation energy, TAFF, Upper critical magnetic field, coherence length

*Corresponding Author: Tel./fax:+90.322.3386060/2496/+90.322.3386070

e-mail: ozcelik@,cu.edu.tr 


\section{Introduction}

Since Bi-based superconductors $\mathrm{Bi}_{2} \mathrm{Sr}_{2} \mathrm{Ca}_{n-1} \mathrm{Cu}_{n} \mathrm{O}_{x}(n=1,2,3)$ were discovered by Maeda et al. [1] many substitutions have been made in BSCCO system in order to improve their physical and magnetic properties [2-5]. The interest in both, the fundamental research and applications in technology, in the last decades increase day by day owing to the fact that BSCCO is the most promising material for potential industrial applications such as superconducting wires, tapes, and thin films [6]. High- $T_{c}$ Bi-based superconductor family is characterized by a high superconducting transition temperature $T c \sim 90$ and $110 \mathrm{~K}$ for the phases with $n=2$, and 3, respectively, and a high upper critical magnetic $B_{c 2}$ which can reach $150 \mathrm{~T}[7]$.

It is rather difficult to realize the mixed state of high-temperature superconductors due to their short coherence length, large anisotropy and thermal energy. The critical current density of a superconductor is a very important parameter for technological applications. Flux pinning mechanism determines the maximum current density that can be driven through a superconductor without dissipation. Flux motion is a mean of dissipating energy in superconductors which was first predicted by Anderson [8]. After this prediction of flux creep, large amounts of research have been recently addressed to the effect of applied external magnetic field on resistive transition in order to better understand the mechanism of flux pinning and flux motion [9-15]. There are several models for understanding the resistivity broadening under a magnetic field, such as thermally activated flux creep [11], flux flow [10], flux line melting and flux cutting [16], etc. The flux line can be thermally activated over the pinning energy barrier, even if the Lorentz force exerted on the flux bundle by the current is smaller than the pinning force. Some researchers have pointed out that a thermally activated flux creep model can describe the data quite well for resistivity region near $T_{C}(\rho=0)[13,17$ 20].

In addition, the material preparation technique plays a crucial role, as important as the substitutions, creating pinning centers in BSCCO systems. Moreover, these $\mathrm{HT}_{\mathrm{C}}$ systems possess a high crystallographic anisotropy which can be exploited by an adequate grain alignment to produce high quality tapes and wires, as well as bulk materials with improved electrical properties. In order to easily obtain very homogeneous samples, solution methods have been shown very adequate and among them, one of best ones is the polymer matrix route [21-26].

In previous studies the structural, physical and superconducting properties of K-substituted BSCCO samples have been studied. In the present work, the effects of $\mathrm{K}$ substitution for $\mathrm{Ca}$ 
on the irreversibility field, upper critical magnetic field, coherence length and activation energies have been investigated. Moreover, the thermally activated flux creep model has been applied on the resistivity curves under various applied magnetic fields between 0-9 T.

\section{Experimental details}

$\mathrm{Bi}_{2} \mathrm{Sr}_{2} \mathrm{Ca}_{1-\mathrm{x}} \mathrm{K}_{\mathrm{x}} \mathrm{Cu}_{2} \mathrm{O}_{\mathrm{y}}$ samples, with $\mathrm{x}=0,0.05,0.075$ and $0.1 \mathrm{~K}$ substitutions have been prepared, using a polymer matrix route from $\mathrm{Bi}\left(\mathrm{CH}_{3} \mathrm{COO}\right)_{2}(\geq 99.99 \%$, Aldrich), $\mathrm{Sr}\left(\mathrm{CH}_{3} \mathrm{COO}\right)_{2} \bullet 0.5 \mathrm{H}_{2} \mathrm{O} \quad(99 \%$, Panreac $), \quad \mathrm{Ca}\left(\mathrm{CH}_{3} \mathrm{COO}\right)_{2} \cdot 2 \mathrm{H}_{2} \mathrm{O} \quad(98 \%$, Alfa Aesar $)$, $\mathrm{Cu}\left(\mathrm{CH}_{3} \mathrm{COO}\right)_{2} \cdot \mathrm{H}_{2} \mathrm{O}$ (98\%, Panreac) and $\mathrm{Co}\left(\mathrm{CH}_{3} \mathrm{COO}\right)_{2} \cdot \mathrm{H}_{2} \mathrm{O}$ (99.9\%, Alfa Aesar) commercial powders. They were weighed in stoichiometric amounts and dissolved in a mixture of glacial acetic acid $\left(\mathrm{CH}_{3} \mathrm{COOH}\right)$ (Panreac PA) and distilled water. Once obtained a clear blue solution, polyethylenimine (PEI) (Aldrich, $50 \mathrm{wt} \%$ water) solution in distilled water, in order to reduce the viscosity of commercial PEI, was added. The mixture becomes dark blue immediately due to the formation of $\mathrm{Cu}-\mathrm{N}$ coordination bonds. The solution was then introduced into a rotary evaporator to reduce its volume $(\sim 80 \%)$ followed by heating on a hot plate at about $100{ }^{\circ} \mathrm{C}$ for total solvent evaporation, producing a thermoplastic dark blue paste. Further heating at around $350{ }^{\circ} \mathrm{C}$ produces a decomposition step. The resulting powder was then milled in an agate mortar and calcined twice at 750 and $800^{\circ} \mathrm{C}$ for $12 \mathrm{~h}$ in order to decompose the alkaline-earth carbonates. Finally, the prereacted homogeneous powders were pressed into pellets with $13 \mathrm{~mm}$ in diameter, and thermally treated in order to produce the Bi2212 superconducting phase. This process was performed under air, and consisted in two steps: $60 \mathrm{~h}$ at $860{ }^{\circ} \mathrm{C}$, followed by $12 \mathrm{~h}$ at $800{ }^{\circ} \mathrm{C}$ and, finally, quenched in air to room temperature.

Magnetoresistivity and magnetic measurements were carried out in a Quantum Design PPMS system. Upper critical magnetic field $\left(H_{c 2}\right)$ values of the samples were calculated from the RT curves. The samples $\mathrm{Bi}_{2} \mathrm{Sr}_{2} \mathrm{Ca}_{1-\mathrm{x}} \mathrm{K}_{\mathrm{x}} \mathrm{Cu}_{2} \mathrm{O}_{8+\mathrm{y}}$ samples, with $\mathrm{x}=0,0.05,0.075$, and 0.1 will be hereafter named as A, B, C and D, respectively

\section{Results and discussion}

\subsection{Magnetoresistivity Measurements}

For the investigation the effect of K-doping on the superconducting properties of the samples, the magnetoresistivity experiments have been performed on the samples in the range of $150 \mathrm{~K}$ 
down to $20 \mathrm{~K}$, for various magnetic fields between 0 and 9 T. In Figure 1, the field dependent resistivity of the samples A, B, C and D as a function of temperature is presented. The onset $\left(T_{c}^{\text {onset }}\right)$ and offset $\left(T_{c}^{\text {offset }}\right)$ temperatures concerning the transition of isolated grains to the superconducting state and to the volume fraction of Bi-2212 phase and/or characteristic of intergranular component, respectively, are summarized in Table 1 . The $T_{c}^{\text {onset }}$ values are deduced from the crossing point of the linear fit of the highest slope and the metallic high temperature part of the $\rho(T)$ curve for each sample. It can be seen that the onset transition temperature $\left(T_{c}^{\text {onset }}\right)$ is above $90 \mathrm{~K}$ pointing out the high- $T c$ Bi-2212 phase is the dominant in the samples. The entire samples exhibit metallic behavior down to $T_{c}^{\text {onset }}$ and the resistivity linearly decreases with decreasing temperature. It is evident from the results displayed in Table 1 that at zero magnetic field both $T_{c}^{\text {onset }}$ and $T_{c}^{\text {offset }}$ values diminish from $91.43 \mathrm{~K}$ to $90.70 \mathrm{~K}, 80.01 \mathrm{~K}$, and $76.76 \mathrm{~K}$ with raising $\mathrm{K}$-content in the samples. This decrease in the temperature values is originated due to the decrement in the number of charge carriers in the $\mathrm{CuO}_{2}$ planes, together with the impurity-induced pair breaking [27-29] or the excess of oxygen-content caused by the substitution $[28,30]$.

While the applied magnetic field increases, slight shifts in the $T_{c}^{\text {onset }}$ are observed. This is probably associated with small changes between the normal and the superconducting resistivity meaning that only a small fraction of the carriers are superconducting [31]. Also, this behavior could be attributed to the strong intragrain pinning energy not allowing any vortex motion in the vicinity of $T c$ [13]. On the other hand, while the temperature lowers, the electrical resistivity becomes more sensitive to the applied magnetic field and the resistivity tail appears at lower temperatures. This is a clear indication that at lower temperatures more charge carriers become superconducting, so the change between the resistivity measured at zero and the applied field becomes greater [32], and weak Josephson type links concerning the grain-boundaries or intergranular effects should be considered [33]. It is very important to emphasize that the zero resistivity temperature is strongly affected by the applied magnetic field revealing a shift to considerably lower temperature as the applied magnetic field increases.

\subsection{Irreversibility Line and Upper Critical Field}

The $I L$ is one of the basic properties of HTSC, fundamentally displaying the change from the flux pinning dominated regime to that dominated by the thermally activated flux flow (TAFF), where resistance appears. The irreversibility field, $H_{i r r}$, has been defined from [34] and plotted in Figure 3a. Plotting the irreversibility limits $H_{\text {irr }}$ of the samples for various 
applied fields in an $H$ - $T$ diagram generates an IL that separates the $H-T$ plane into two regions: a low temperature one, in which the flux dynamics is dissipative and thus irreversible, and a high temperature one, where the flux motion is free and magnetically reversible. As seen in Figure $3 \mathrm{a}, H_{\text {irr }}$ increases and IL shifts to higher temperatures up to $\mathrm{x}=0.075$, then it decreases with increasing K-content. These results indicate that the strength vortex fluctuations are getting stronger with increasing K-content. The graph of upper critical magnetic fields $H_{c 2}(T)$ versus temperature $T c$ for all the samples is shown in Figure $3 \mathrm{~b}$. It has been found that $H_{c 2}(T)$ values increase with decreasing temperature. $H_{C 2}(0)$ is the upper critical magnetic field at the temperature $T=0 \mathrm{~K}$ and can be estimated by using WerthomerHelfond-Hohenberg (WHH) formula [35]:

$$
H_{C 2}(0)=-0.693 T c\left(\frac{d L e 2(T)}{d T}\right)_{T c}
$$

Using $T_{C}$ values determined from the midpoint of the linear fit of the highest slope of the resistivity curves at zero field given in Figure $1, H_{C 2}(0)$ values have been calculated and plotted in Figure 4. Initial substitution of K-content up to $\mathrm{x}=0.075$ increases the $H_{\mathrm{C} 2}(0)$ value but further substitution lowers it. Calculated $H_{C 2}(0)$ values are 62, 72, 75, and 53 Tesla for the samples A, B, C, and D, respectively.

The coherence length $\xi$ is one of the characteristic parameter of the superconductors. The coherence length $\xi$ is often calculated from the equation,

$$
H_{a 2}(0)=\Phi_{U} / 2 \pi \xi^{2}
$$

where $H_{c 2}(0)$ is the upper critical magnetic field at the temperature $T=0 \mathrm{~K}$ and $\Phi_{\mathrm{U}}$ is the magnetic field flux quantum $\left(2.07 \times 10^{-15} \mathrm{Tm}^{2}\right)$ [9,14,34,36,37]. By using Eq.2, the coherence lengths of the samples at $T=0 \mathrm{~K}$ have been calculated and displayed in Figure 4. As it is seen that $\xi(0)$ varied from 23, 21, 20, and 25, for the samples A, B, C and D, respectively. Similar results are reported for the coherence length $\xi(0)$ and $H_{c 2}(0)$ values for Bi-based superconductors [14].

\subsection{Activation Energy}

It is well established that the resistive transition broadening under magnetic fields can be interpreted to be a result of TAFF in the vortex liquid state [6-9] where the vortex system is in a liquid state with no long-range order and vanishing shear modulus immediately below $T c(H)$. The pinning potential hindering the free flow of vortices is caused by a combination of point-like defects and the correlated defects of the columnar microstructure. As can be seen 
easily in Figure 2, $\ln \left(\rho / \rho_{0}\right)$ vs. 1/T plots at different magnetic fields, for pure and K-substituted materials, show nonlinear behavior. Therefore, according to the thermally activated flux creep model, the dissipated energy (resistivity) in the tail part of the magnetoresistivity plot is defined by an Arrhenius-type equation [8,38]:

$$
\rho(H, T)=\rho_{0} \exp \left[-U(H) / k_{B} T\right]
$$

where $U$ is the flux pinning energy or activation energy for flux creep that depends on the temperature and magnetic field, $\rho_{0}$ is the pre-exponential factor or simply the prefactor, and $k_{B}$ is Boltzmann constant. The activation energy can be directly deduced from the slope of the plot of $\log \left(\rho / \rho_{0}\right)$ versus $1 / T$ as shown in Figure 5, where the value of $\rho_{0}$ at the temperature of $110 \mathrm{~K}$ was used.

Figure 5 demonstrates the calculated activation energy from the linear data in the tail part of the plots, for all the samples. It is evident from this figure that the activation energies of the samples reduce significantly with the enhancement in both the $\mathrm{K}$ doping level and applied magnetic field. This effect can be attributed to the raise of oxygen concentration in the grain boundaries, revealing a reduction of the intergranular coupling and a raise of the amount of weak links. With an increase of the weak links, the vortices creep easily, whereas the pinning energy or activation energy decreases. These results are similar to those previously reported $[9,31,34]$. It is also worthwhile to mention that the main differences between curves in Figure 5 have been observed for fields below 1 Tesla. Since below 1 Tesla, the applied magnetic field has penetrated only the intergranular region, a possible interpretation for the differences between curves can be ascribed to the existence of different superconducting levels within the samples in these regions: the superconducting grains, the weak-links, and the superconducting clusters [39-41]. Thus, it would be reasonable to assure that in sample C, the fraction of grains interconnected by strong-links (clusters) is very large as compared with those of the other samples.

\section{Conclusions}

In summary, polycrystalline $\mathrm{Bi}_{2} \mathrm{Sr}_{2} \mathrm{Ca}_{1-\mathrm{x}} \mathrm{K}_{\mathrm{x}} \mathrm{Cu}_{2} \mathrm{O}_{8+\mathrm{y}}$ superconductors with $\mathrm{x}=0.0,0.05,0.075$, and 0.1 have been prepared by the polymer matrix method. The change of the flux pinning mechanism has been studied using magnetoresistivity measurements. The activation energies, irreversibility fields $\left(H_{i r r}\right)$, upper critical fields $\left(H_{C 2}\right)$ and coherence lengths at $0 \mathrm{~K}(\zeta(0))$ were evaluated from the resistivity versus temperature $(\rho-T)$ curves, under DC magnetic fields up to $9 \mathrm{~T}$. The electrical resistivity broadening behavior in the magnetic field has been studied using 
the thermally activated flux creep model. According to this model, initial substitution of $\mathrm{K}$ up to $x=0.075$ increases the flux pinning energy, $U$, which indicates higher slope in the energy barriers. It is possible to say that the raise of the energy barriers may arise from the decreasing of the weak links which, in turn, makes that the vortices does not creep easily and the pinning energy or activation energy increases since the strong pinning centers develop in the system by $\mathrm{K}$ addition.

\section{Acknowledgement}

This work is supported by Research Fund of Cukurova University, Adana, Turkey, under grant contracts no: FEF 2013YL17 and FEF2013BAP22. 


\section{References}

[1] H.Maeda, Y. Tanaka, M. Fukutomi, T. Asano, Jpn. J. Appl. Phys. 27, L 209 (1988)

[2] B.Chattopadhyay, B.Bandyopadhyay, A.Poddar, P.Mandal, A.N.Das, B.Ghosh, Physica C $331,38(2000)$

[3] G.Ilonca, V.Toma, T.R.Yang, A.V.Pop, P.Balint, M. Bodea, E.Macocian, Physica C 369, 460 (2007)

[4] X.L.Wang, J.Horvat, G.D.Gu, K.K.Uprety, H.K.Liu, S.X.Dou, Physica C 337, 221 (2000)

[5] T.Yamamoto, I.Kakeya, K.Kadowaki, Physica C 460-462, 799 (2007)

[6] M. Cyrot, D. Pavuna, Introduction to Superconductivity and High-Tc Materials, p. 249. World Scientific, Singapore (1995)

[7] G. Blatter, M.V. Feigelman, V.B. Ceshkenbein, A.I. Larkin, V.M. Vinokur, Rev. Mod. Phys. 6, 1125 (1994)

[8] P.W. Anderson, Phys.Rev.Lett. 9,309 (1962)

[9] M.Erdem, O.Ozturk, E.Yucel, S.P.Altintas, A.Varilci, C.Terzioğlu, I.Belenli, Physica B 406, 705 (2011)

[10] M.Tinkham, Phys. Rev. Lett. 61, 1658 (1988)

[11] T.T.Palstra, B.Batlogg, R.B. Van Dover, L.F. Scheemeyer, J. V.Waszczak, Appl. Phys. Lett. 54, 763 (1989)

[12] D.H. Kim, K. F. Gray, R. T. Kampwirth, D. M. Mckay: Phys, Rev. B 42,6249 (1990)

[13] B.Özkurt, B.Özçelik, J. Low Temp.Phys. 156, 22 (2009)

[14] D.Yazici, M.Erdem, B.Özçelik, J. Supercond. Nov.Magn. 25, 1811 (2012)

[15] D. Sharma, R. Kumar, V.P.S. Awana, Solid State Commun. 152, 941 (2012)

[16] M.R.Mohammadizadeh, M.Akvahan, Physica C 390, 134 (2003)

[17] T.T. Palstra, B. Batlogg, L.F. Schneemeyer, J.V. Waszczak, Phys. Rev. Lett. 61 1662 (1988).

[18] A.P. Malozemoff, T.K. Worthington, E. Zeldov, N.C. Yeh, M.W. McElfresh, in Strong Correlation and Superconductivity, ed. by H. Fukuyama, S. Maekawa, A.P. Malozemoff. Springer Series in Sol.State Sci., vol. 89, Springer, Berlin, (1989)

[19] R.Griessen, Phys. Rev. Lett. 64, 1674 (1990)

[20] R.C. Ma, et al., Physica C 405, 34 (2004)

[21] G. F. de la Fuente, A. Sotelo, Y. Huang, M. T. Ruiz, A. Badia, L. A. Angurel, F. Lera, R. Navarro, C. Rillo, R. Ibañez, D. Beltran, F. Sapiña, A. Beltran, Physica C, 185, 509 (1991) 
[22] B. Özçelik, B. Özkurt, M.E. Yakıncı, A. Sotelo, M. A. Madre, J. Supercond. Nov. Magn., 26, 873 (2013)

[23] A.Sotelo, H.Szillat, P. Majewski, F. Aldinger, Supercond. Sci. Technol. 10, 717-720 (1997)

[24] H. Gündogmus, B. Özçelik, B. Özkurt, A. Sotelo, M.A. Madre, J. Supercond. Nov. Magn, 26, 111(2013)

[25] A. Ozaslan, B. Özçelik, B. Özkurt, A. Sotelo, M.A. Madre, J. Supercond. Nov. Magn, 27, 53 (2014)

[26] C. Kaya, B. Özçelik, B. Özkurt, A. Sotelo, M.A. Madre, J Mater Sci:Mater Electron, 24, $1580(2013)$

[27] A. Maeda, T. Yabe, S. Takebayashi, M. Hase, K. Uchinokura, Phys. Rev. B41, 4112 (1990)

[28] S. T. Lin, W. S. Clung, C. Y. Chou, and C. hl. Lin, J. Phys. Condens. Matter 2, 8763 (1990)

[29] P. Sumana Prabhu, M. S. Ramachandra Rao, and G. V. Subba Rao, Physica C211, 279 (1993)

[30] M. Boekholt, Th. Eilmeier, L. Buschmann, M. Fleuster, G. Giintherodt, Physica C198, 33, (1992)

[31] D. Yazici, M. Erdem, B. Ozcelik, J Supercond Nov Magn, 25, 725 (2012)

[32] N.G. Gomaa, Egypt. J. Solid 22, 256, (1999)

[33] A.R Jurelo, J.V Kunzler, J.Schaf, P.Pureur, J.Rosenblatt, Phys. Rev. B 5614815 (1997)

[34] H. Gündogmus, B. Özçelik, A. Sotelo, M.A. Madre, J Mater Sci: Mater Electron 24, $2568(2013)$

[35] N. R. Werthamer, E. Helfand, P. C. Hohenberg, Phys. Rev., 147295 (1966)

[36] H.C. Yang, L.M. Wang, Phys. Rev. B 59, 13 (1999)

[37] B. Özçelik, H. Gündogmus, D. Yazıcı, J Mater Sci: Mater Electron., 25, 2456, (2014)

[38] P.W. Anderson, Y.B. Kim, Rev. Mod. Phys. 36, 39, (1964)

[39] E.Govea-Alcaide,I.Garcia-Fornaris, P.Mune, and R.F.Jardim, Eur.Phys.J.B 58, 373, (2007)

[40] P. Kameli, H. Salamati, I. Abdolhosseini, D. Sohrabi, Physica C 468, 137, (2008)

[41] D. Yazıcı, B. Özçelik, M. E. Yakıncı, J Low Temp Phys 163, 370, (2011) 
Table.1. $T_{c}^{\text {onset }}$ and $T_{c}^{\text {offset }}$ values of the samples at various applied magnetic field

\begin{tabular}{|c|c|c|c|c|c|c|}
\hline Sample & OTesla & 1Tesla & 3Tesla & 5Tesla & 7Tesla & 9Tesla \\
\hline 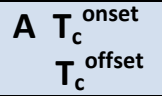 & $\begin{array}{l}91.43 \mathrm{~K} \\
80.01 \mathrm{~K}\end{array}$ & $\begin{array}{l}91.33 \mathrm{~K} \\
64.06 \mathrm{~K}\end{array}$ & $\begin{array}{l}90.70 \mathrm{~K} \\
54.78 \mathrm{~K}\end{array}$ & $\begin{array}{l}90.60 \mathrm{~K} \\
48.48 \mathrm{~K}\end{array}$ & $\begin{array}{l}90.32 \mathrm{~K} \\
44.59 \mathrm{~K}\end{array}$ & $\begin{array}{l}90.23 \mathrm{~K} \\
42.00 \mathrm{~K}\end{array}$ \\
\hline $\begin{array}{c}\text { B } T_{c}^{\text {onset }} \\
T_{c .}{ }^{\text {offset }}\end{array}$ & $\begin{array}{l}91.40 \mathrm{~K} \\
80.13 \mathrm{~K} \\
\end{array}$ & $\begin{array}{l}91.18 \mathrm{~K} \\
66.90 \mathrm{~K} \\
\end{array}$ & $\begin{array}{l}90.55 \mathrm{~K} \\
58.52 \mathrm{~K} \\
\end{array}$ & $\begin{array}{l}90.07 \mathrm{~K} \\
53.48 \mathrm{~K} \\
\end{array}$ & $\begin{array}{l}90.01 \mathrm{~K} \\
49.64 \mathrm{~K} \\
\end{array}$ & $\begin{array}{l}89.89 \mathrm{~K} \\
46.52 \mathrm{~K} \\
\end{array}$ \\
\hline $\begin{array}{c}C T_{c}{ }_{c}{ }^{\text {onset }} \\
T_{c .}{ }^{\text {offset }}\end{array}$ & $\begin{array}{l}91.36 \mathrm{~K} \\
82.38 \mathrm{~K}\end{array}$ & $\begin{array}{l}91.15 \mathrm{~K} \\
68.43 \mathrm{~K}\end{array}$ & $\begin{array}{l}90.44 \mathrm{~K} \\
59.49 \mathrm{~K}\end{array}$ & $\begin{array}{l}89.98 \mathrm{~K} \\
54.56 \mathrm{~K}\end{array}$ & $\begin{array}{l}89.68 \mathrm{~K} \\
50.50 \mathrm{~K}\end{array}$ & $\begin{array}{l}89.33 \mathrm{~K} \\
47.70 \mathrm{~K}\end{array}$ \\
\hline $\begin{array}{c}\text { D } T_{c .}^{\text {onset }} \\
T_{c .}{ }^{\text {offset }}\end{array}$ & $\begin{array}{l}90.77 \mathrm{~K} \\
76.76 \mathrm{~K}\end{array}$ & $\begin{array}{l}90.28 \mathrm{~K} \\
61.63 \mathrm{~K}\end{array}$ & $\begin{array}{l}89.70 \mathrm{~K} \\
52.56 \mathrm{~K}\end{array}$ & $\begin{array}{l}89.10 \mathrm{~K} \\
47.75 \mathrm{~K}\end{array}$ & $\begin{array}{l}88.9 \mathrm{~K} \\
44.32 \mathrm{~K}\end{array}$ & $\begin{array}{l}88.6 \mathrm{~K} \\
41.69 \mathrm{~K}\end{array}$ \\
\hline
\end{tabular}




\section{Figure captions}

Figure 1. Temperature dependence of resistivity for various applied magnetic fields for samples A, B, C and D

Figure 2. Arrhenius plot of the resistivity of samples A, B, C and D

Figure 3. Irreversibility field, $H_{i r r}$, and Upper critical magnetic field $H_{c 2}(T)$ versus $\mathrm{K}$ content.

Figure 4. Variation of the upper critical magnetic field at a temperature $T=0 \mathrm{~K}, H_{C 2}(0)$, and coherence length, $\zeta$, values versus K-content.

Figure 5. Flux pinning energy $U$ versus applied magnetic field for samples A,B,C and D. 
Figure 1
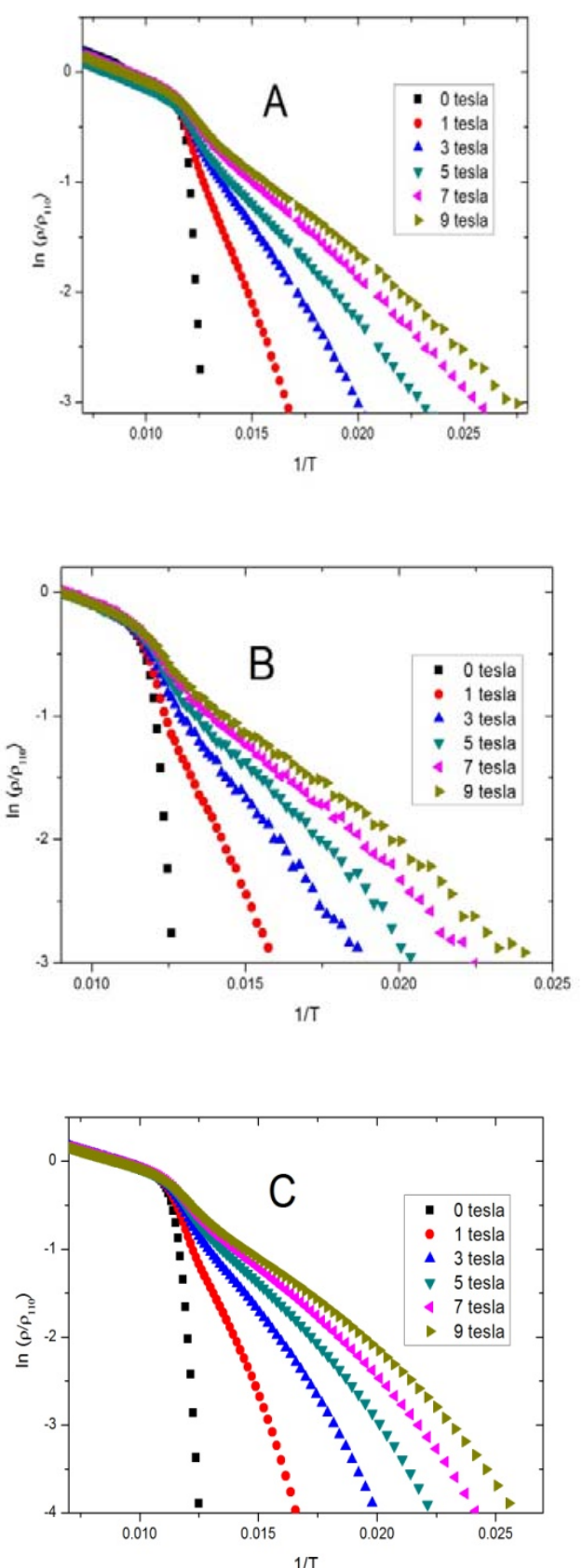
Figure 2

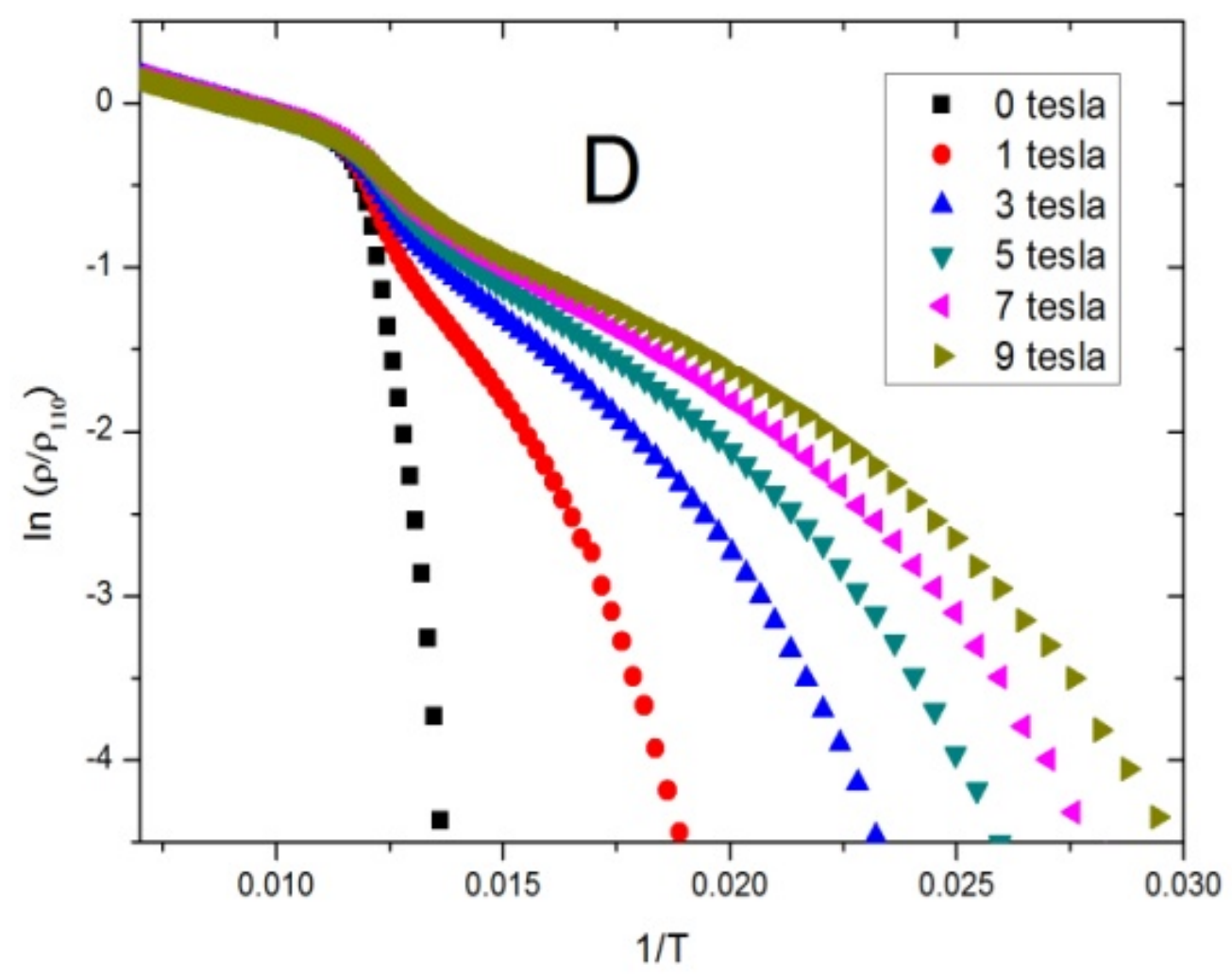


Figure 3
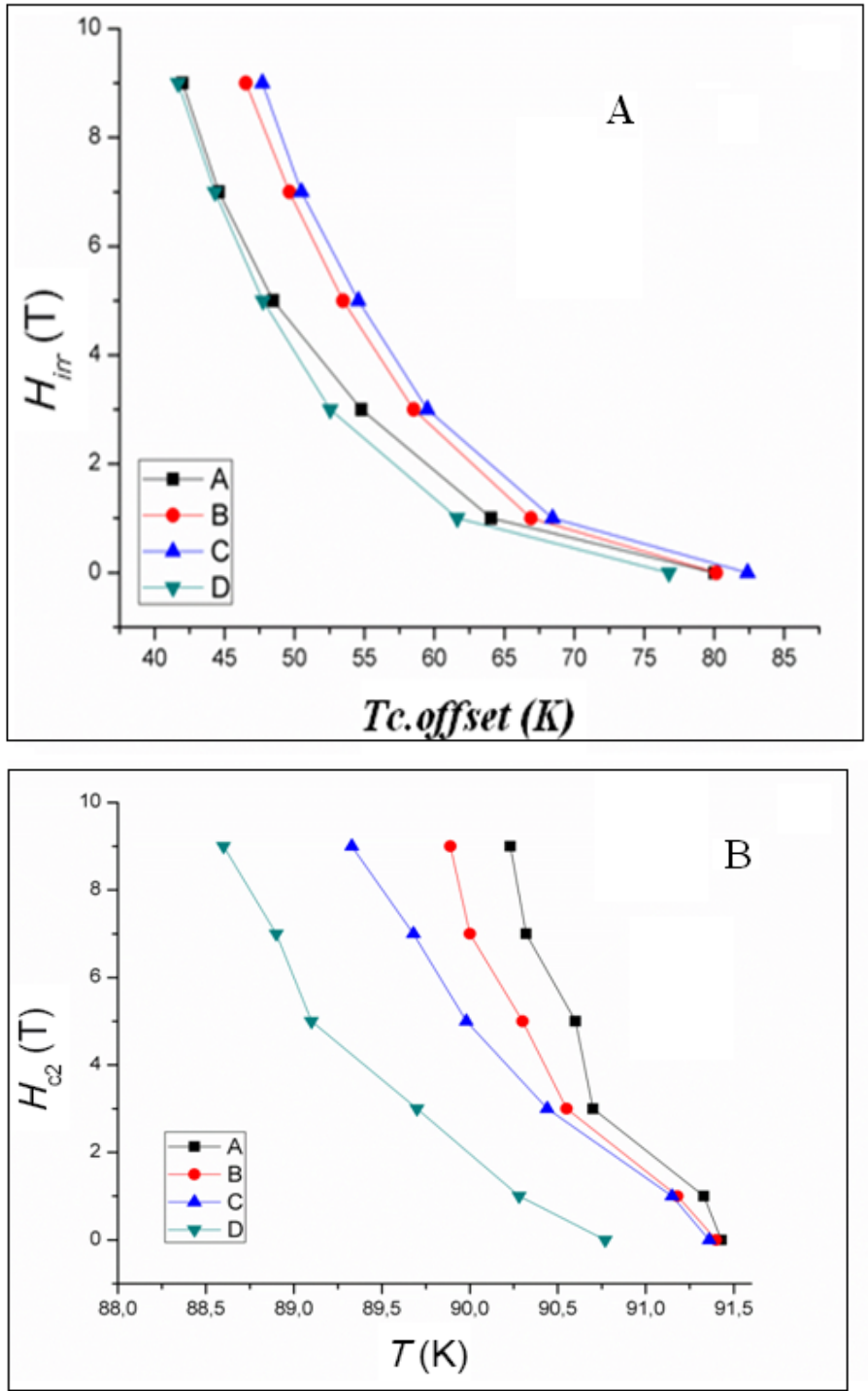
Figure 4

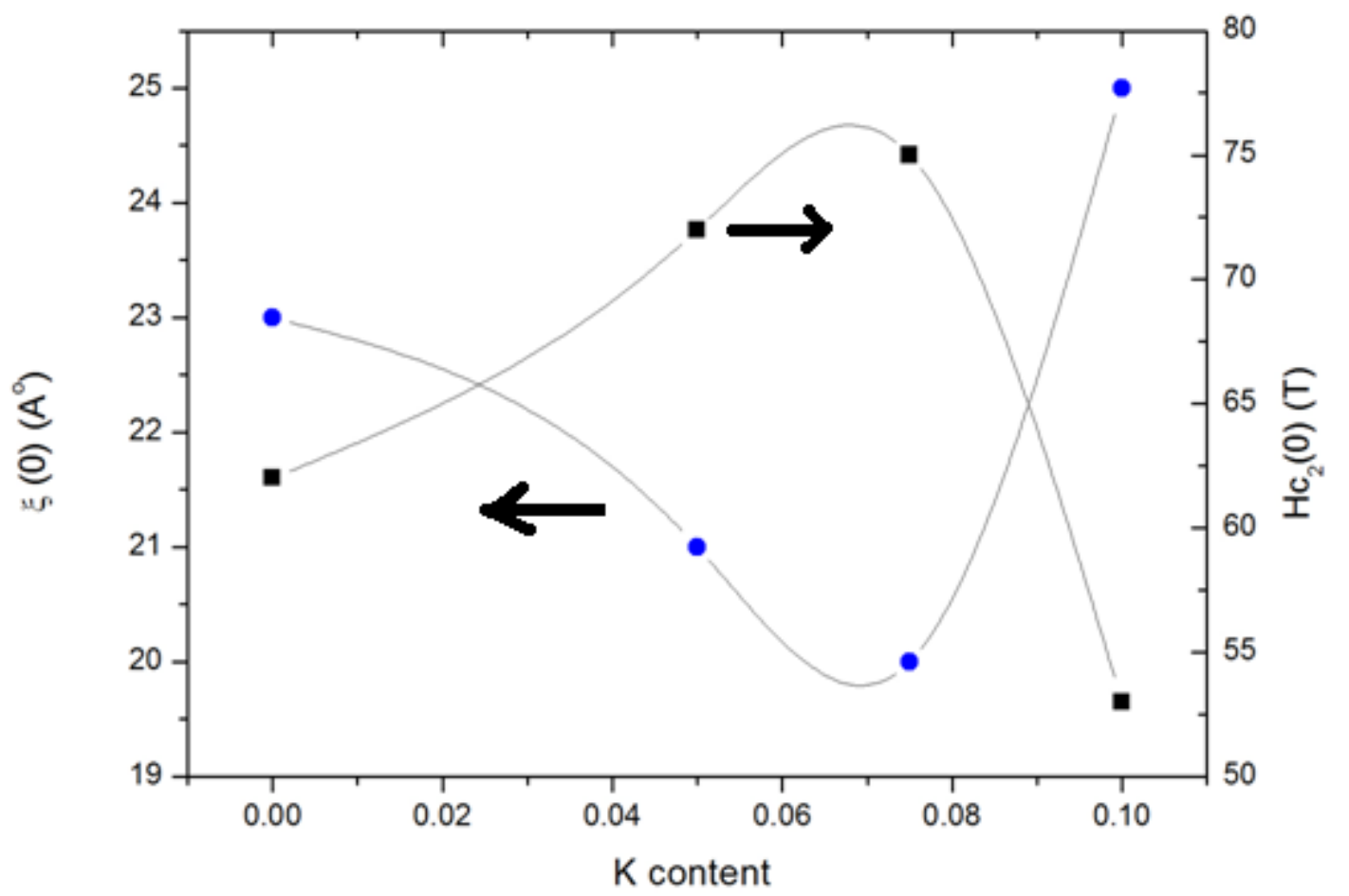


Figure 5

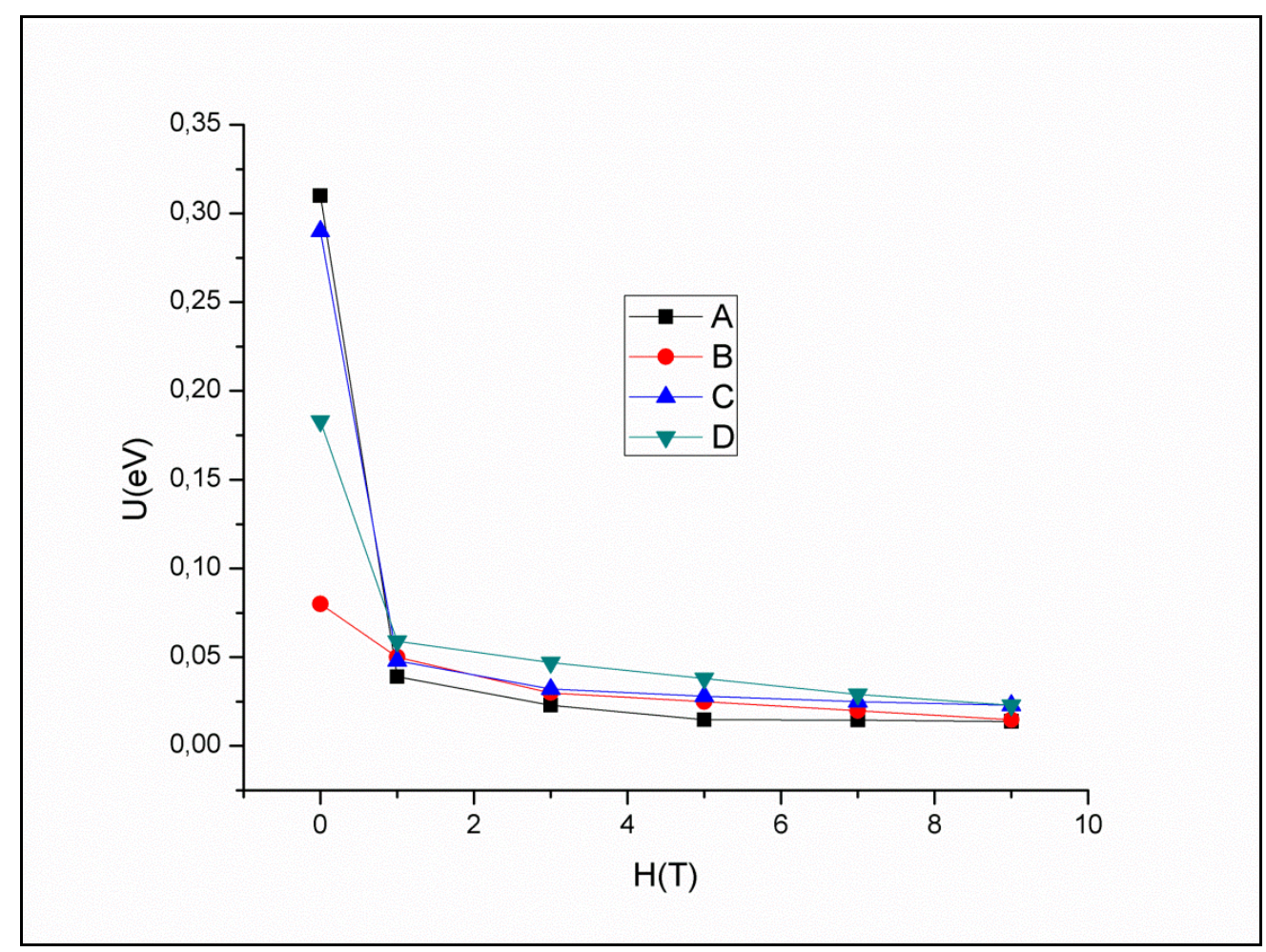

